\title{
A Feasibility Study for Life Signs Monitoring via a Continuous-Wave Radar
}

\author{
Francesco Soldovieri, ${ }^{1}$ Ilaria Catapano, ${ }^{1}$ Lorenzo Crocco, $^{1}$ \\ Lesya N. Anishchenko, ${ }^{2}$ and Sergey I. Ivashov ${ }^{2}$ \\ ${ }^{1}$ Istituto per il Rilevamento Elettromagnetico dell'Ambiente, Consiglio Nazionale delle Ricerche, Via Diocleziano 328, \\ 80124 Napoli, Italy \\ ${ }^{2}$ RSLab, Bauman Moscow State Technical University, 2nd Baumanskaya 5, Moscow 105005, Russia
}

Correspondence should be addressed to Francesco Soldovieri, soldovieri.f@irea.cnr.it

Received 7 June 2012; Accepted 12 August 2012

Academic Editor: Danilo Erricolo

Copyright (C) 2012 Francesco Soldovieri et al. This is an open access article distributed under the Creative Commons Attribution License, which permits unrestricted use, distribution, and reproduction in any medium, provided the original work is properly cited.

We present a feasibility study for life signs detection using a continuous-wave radar working in the band around $4 \mathrm{GHz}$. The data-processing is carried out by using two different data processing approaches, which are compared about the possibility to characterize the frequency behaviour of the breathing and heartbeat activity. The two approaches are used with the main aim to show the possibility of monitoring the vital signs activity in an accurate and reliable way.

\section{Introduction}

The remote and contactless detection and monitoring of life movements and signs as breathing and heartbeat activity is a topic of increasing attention in many fields such as the homeland defence and homeland security systems $[1,2]$; the rescue of persons buried under rubble or under snow [3-5]; the medical field for a contactless monitoring of the conditions of patients [6]. Moreover, the use of noncontact microwave-based transceivers has been recently proposed as diagnostic tool in the biomedical field $[4,7,8]$.

In this work, we propose a feasibility study of a life signs detection and characterization system using a multifrequency radar. The measurements are processed by using two different data-processing approaches, whose performance is compared in terms of frequency characterization of breathing and heartbeat activity.

The multifrequency bioradar with a quadrature receiver has been designed at the Remote Sensing Laboratory, Bauman Moscow State Technical University with the aim to carry out remote monitoring of movement activity, breathing, and pulse of human heart [9].

The first data processing approach has been presented in [10] and aims at providing frequency analysis of the life signs activity by maximizing the scalar product of the Fourier transform of the measured signal, accounting for the one researched for displacement, and the signal given by a theoretical electromagnetic model. The second approach aims at providing information not only about the frequency of life signs but also at gaining information about the range of the investigated target by getting range-frequency matrix. Separation of respiration and heartbeat signals was made by application of rejection filtration to corresponding line of range-frequency matrix $[9,11]$.

The paper is organised as follows. Section 2 is devoted to describe the multifrequency radar system and to give details about the experiment considered later for the data processing. Section 3 gives the description of the first data-processing approach, whereas the second reconstruction approach is presented in Section 4. The results of the experiment are shown in Section 5 where a comparison between the two data-processing approaches is discussed. Finally, conclusions follow.

\section{Experimental Setup and Experiment Description}

This section is devoted to give a brief description of the multifrequency radar system as well as of the configuration 


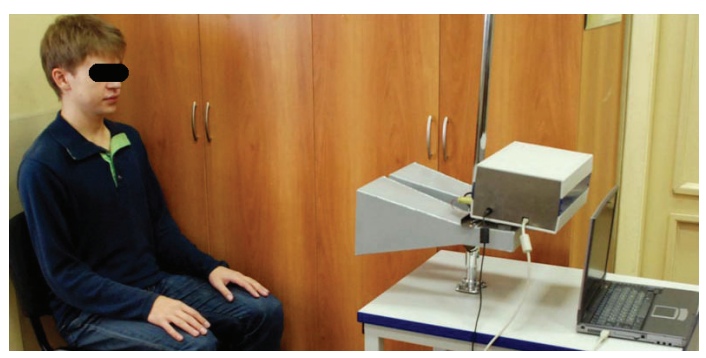

Figure 1: Sketch of the experimental setup.

TABLE 1: Parameters of the radar system.

\begin{tabular}{lc}
\hline Number of frequencies & 16 \\
Sampling frequency & $62.5 \mathrm{~Hz}$ \\
Operating frequency band & (time spacing $0.016 \mathrm{sec}$ ) \\
Distance space resolution & $3.6-4.0 \mathrm{GHz}$ \\
Dynamic range of the recording signals & $0.5 \mathrm{~m}$ \\
Recording signals band & $60 \mathrm{~dB}$ \\
Dimensions of antennas block & $0.03-5 \mathrm{~Hz}$ \\
\hline
\end{tabular}

deployed for the measurements and to provide details about the acquisition of the two datasets processed in the sections below.

A multifrequency radar designed at the Remote Sensing Laboratory (Bauman Moscow State Technical University) was used in the experiment $[9,11]$. The radar has the main technical parameters reported in Table 1. In particular, it operates by emitting and collecting a field in a working frequencies range from $3.6 \mathrm{GHz}$ to $4 \mathrm{GHz}$ and this entails a spatial resolution, related to the operating frequency band, approximately equal to $0.5 \mathrm{~m}$.

Figure 1 is a photo of the experiment where the subject is located in front of the radar system. In particular, the experiment was carried out with a male of 20 years old, no bad habits, and professional skier; the distance between antennas and subject was $1 \mathrm{~m}$. The experiment was divided into two stages. During the first stage, monitoring of breathing and pulse parameters at steady state was carried out, and it took about 5 minutes. At the second stage a breath-holding test was carried out. It gives a rough index of cardiopulmonary reserve, measured by the length of time a person can hold breath. The test is widely known in medicine and is used for estimating fitness of the human body while training of pilots, submariners, and divers. Each stage of the experiment was carried out for several times for slightly different displacement of examinee and bioradar in order to confirm the possibility of multifrequency bioradar to estimate the range by using the second data-processing approach.

\section{The First Data Processing Approach}

As said above, the problem at hand is concerned with the detection of vital signs (breathing and heartbeat) and the determination of their frequency for the case of human being in free space.

To this end, we adopt a very simple model of the electromagnetic scattering of a vibrating metallic (perfectly electric conducting) plate located in free space. The metallic plate is located at a distance $z_{0}$ from the antenna system, and accordingly, its time-varying position is given as $z(t)=z_{0}+$ $A \sin \left(\bar{\omega}_{D} t\right)$, where $A$ is maximum displacement with respect to the rest position $z_{0}$, and $\bar{\omega}_{D}$ is the unknown Doppler frequency. To compute the field reflected by the vibrating plate, we exploit the hypothesis of quasistationarity: we "freeze" the plate at each time $t$ when it occupies the position $\hat{z}$ and compute the field as if it were stationary.

If we assume the electromagnetic field as a plane wave with propagation direction along the $z$-axis, the field collected by the antenna system is given as

$$
E_{R}=E_{0} \exp \left(-2 j k_{0}\left(z_{0}+A \sin \left(\bar{\omega}_{D} t\right)\right)\right)+E_{\text {clut }},
$$

where $k_{0}=2 \pi / \lambda$ (being $\lambda$ the wavelength in free space), and $E_{\text {clut }}$ is due to the static clutter in absence of the plate, that is, it accounts for the scattering from static objects. Thus, the problem at hand is stated as the estimation of the Doppler frequency $\bar{\omega}_{D}$ starting from the knowledge of the reflected field measured over a finite time interval $[0, T]$.

The proposed reconstruction procedure is based on three different steps. The first one is concerned with removal/mitigation of the static clutter, that is, the $E_{\text {clut }}$ term in (1). The ideal clutter removal strategy would be based on the difference between the actual signal and the one when no vital signs are present (background signal). Since such a background measurement is not available at all, the necessity of alternative strategies arises. In this paper, the static clutter removal is carried out by the following steps: first, we compute the mean value $E_{\text {mean }}$ of the signal over the interval domain $[0, \mathrm{~T}]$; then, we subtract $E_{\text {mean }}$ to the measured one $E_{R}(t)$ to achieve $\widetilde{E}_{R}(t)=E_{R}(t)-E_{\text {mean }}$. The subsequent processing is then performed on $\widetilde{E}_{R}(t)$.

After a Fourier transform is performed on the resulting signal so to compute the function $G\left(\omega_{D}\right)$ in Doppler domain. First, we compute the Fourier transform of the model signal $\exp \left(-j 2 k_{0} A \sin \left(\omega_{D} t\right)\right)$ as to obtain

$$
\begin{aligned}
E_{\text {model }}\left(\omega_{D}\right)= & \int_{0}^{T} \exp \left(-2 j k_{0} A \sin \left(\bar{\omega}_{D} t\right)\right) \exp \left(-j \omega_{D} t\right) d t \\
= & \int_{0}^{T} \sum_{n=-\infty}^{\infty} J_{-n}\left(2 k_{0} A\right) \exp \left(j n \bar{\omega}_{D} t\right) \exp \left(-j \omega_{D} t\right) d t \\
= & \sum_{n=-\infty}^{\infty} J_{-n}\left(2 k_{0} A\right) \sin c\left[\left(\frac{T}{2}\right)\left(\omega_{D}-n \bar{\omega}_{D}\right)\right] \\
& \times \exp \left(-j\left(\omega_{D}-n \bar{\omega}_{D}\right)\left(\frac{T}{2}\right)\right),
\end{aligned}
$$

where we exploit the well-known Fourier expansion of the term $\exp \left(-j 2 k_{0} A \sin \left(\bar{\omega}_{D} t\right)\right)$, and $J_{n}(\bullet)$ denotes the Bessel function of first kind and $n$th order. Therefore, the Fourier transform $E_{\text {model }}\left(\omega_{D}\right)$ is made up of a train of sinc functions centred at $n \bar{\omega}_{D}$. 
Finally, the unknown Doppler frequency $\bar{\omega}_{D}$ is determined as the quantity that maximizes the scalar product between the modulus of the "measured" Fourier transform $\left|G\left(\omega_{D}\right)\right|^{2}$ and the modulus of the Fourier transform of the model signal $\left|E_{\text {model }}\left(\omega_{D}\right)\right|^{2}$.

It is worth noting that in the above-outlined procedure the maximum displacement $D$ (see (2)) is still unknown. In principle, such a quantity could be determined together with the Doppler frequency to maximize the scalar product. In the cases at hand, in order to make the determination procedure fast to approach realistic cases, we assume an estimate of the maximum displacement as $A=0.5 \mathrm{~cm}$ for the breathing and $A=1 \mathrm{~mm}$ for the heartbeat.

\section{The Second Data-Processing Approach}

The second data-processing approach is designed with the aim to gain information not only about the frequency behavior of the life signs but even about the range of the investigated subject $[9,11]$. The procedure can be summarized according to the step below.

The first step allows to build the range-frequency matrix [9]; this matrix contains all possible signal reflections including ones from the motionless objects (MOs), located in different range cells. These objects are the cause of static clutter. The suppression of signals from MO is carried out by rejection of the matrix components for the approach zero frequencies. The range-frequency matrix resulting from the suppression of the zero or nearly zero frequencies is given in the upper panel of Figure 2.

The separation between the breathing and heartbeat signals is carried out next by using rejection of the frequency components corresponding to breathing in the range-frequency matrix, and the result is shown in the lower panel of Figure 2.

Reconstruction of breathing and heartbeat signals is carried out by applying inverse Fourier transform to the matrix row corresponding to the distance to the examinee $(1.5 \mathrm{~m})$ and evaluating its phase. Thus obtained signals corresponding to range-frequency matrixes from Figure 2 are shown in Figure 3.

Figure 3 points out in a clear way the good performances of the approach in separating breathing and heartbeat signals.

\section{Reconstruction Results}

This section is devoted to present the two reconstruction results for breathing/and heartbeat detection and characterization starting from the measurements described in Section 2. In particular, we show the processing results for the datasets collected at the two stages of the experiment for the illumination frequency equal to $3.6 \mathrm{GHz}$.

For the first stage of the experiment, there was a time window of $304 \mathrm{sec}$. For this overall time window, we have considered 19 time intervals made of 1024 time samples (for a time interval equal to $16,3 \mathrm{sec}$ ), and for each of these intervals, we have applied the two data-processing approaches described in the above sections.

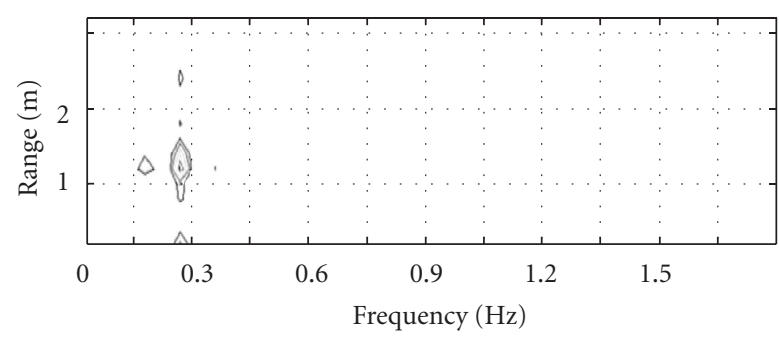

(a)

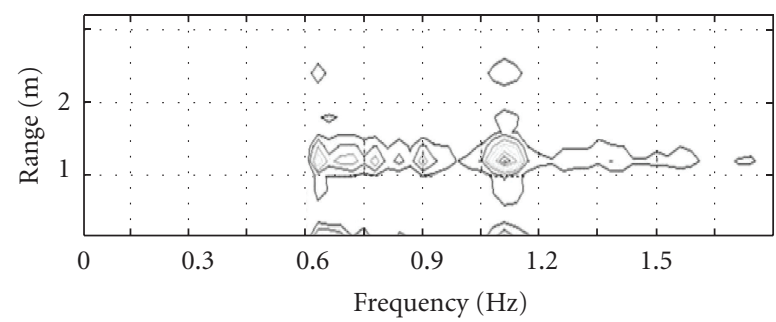

(b)

FIGURE 2: Range-frequency matrix for the examinee at $1.5 \mathrm{~m}$ range: (a) (upper) before breathing harmonics rejection; (b) (lower) after breathing harmonics rejection.

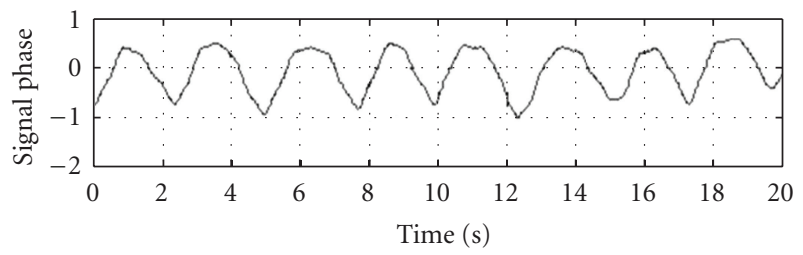

(a)

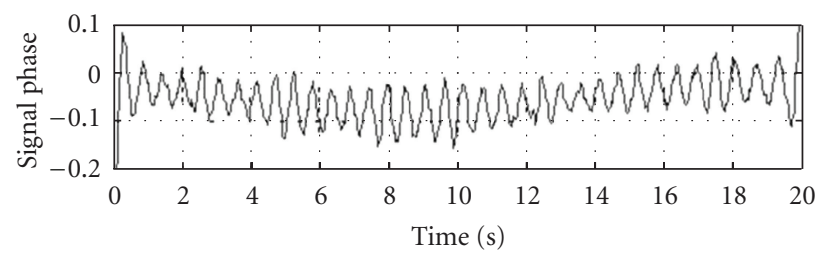

(b)

FIGURE 3: Reconstructed breathing (a) and heartbeat (b) signals of the examinee corresponding to the range-frequency matrices from Figure 2. The signal phase is normalized with respect to its maximum.

Figure 4 depicts the modulus of the signal and also the 19 time intervals analyzed in the monitoring are also pointed out.

The results of the breathing activity monitoring for the two approaches are depicted in Figure 5. Upper and lower panels give the results of the processing achieved by the first and second approachs, respectively.

A good agreement is observed between the results for the two data-processing approaches; in particular, an almost uniform breathing behaviour is observed with a frequency of $18 \mathrm{acts} / \mathrm{min}$ apart from few time intervals.

Figure 6 depicts the heartbeat analysis, upper and lower panels give the results of the processing achieved by the first 


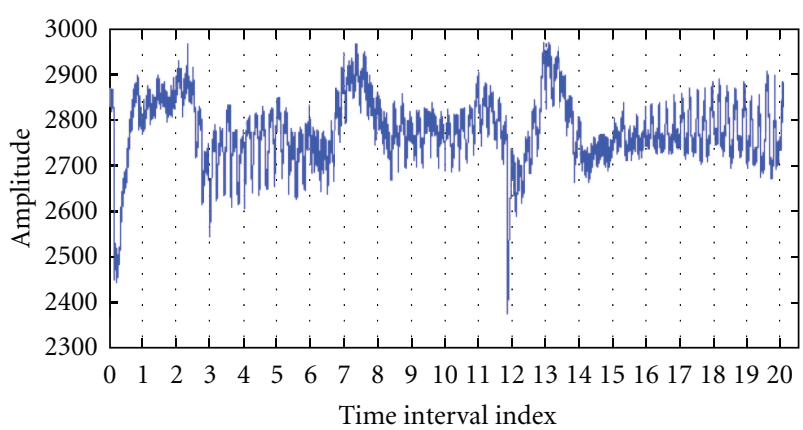

FIgure 4: Amplitude of the life signal. The time intervals used in the monitoring are also pointed out.

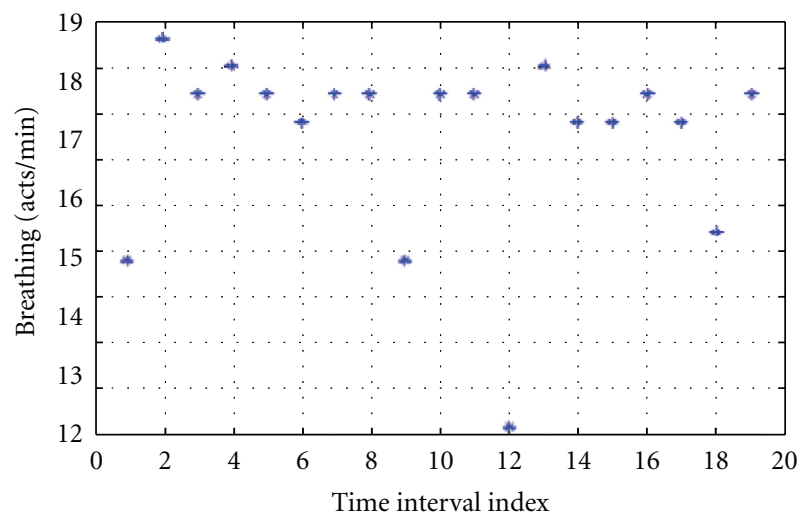

(a)

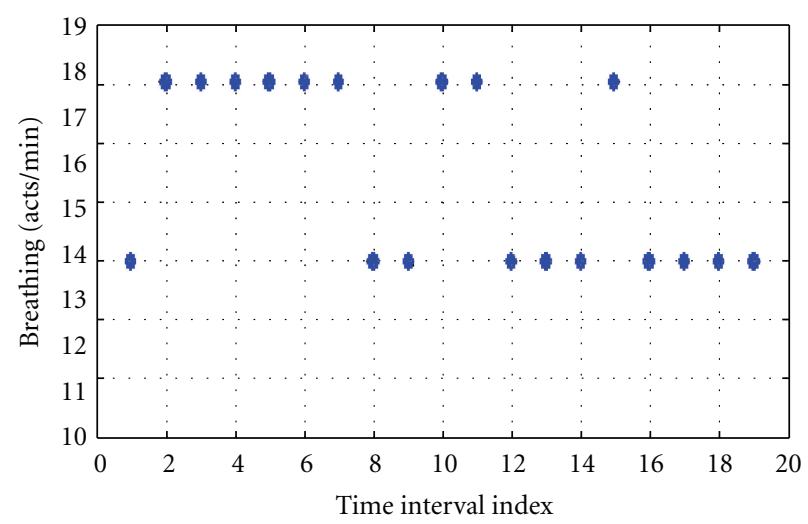

(b)

Figure 5: Comparison between the dataprocessing approaches for the breathing activity monitoring: (a) first data-processing approach; (b) seconddata-processing approach.

and second approachs, respectively. It can be seen that the average frequency is about 80 beats per minute. In addition, we can note a correlation between the time behavior of the breathing and the one of the heart beat. As a matter of fact, when the breathing frequency decreases, the heartbeat frequency decreases too.

The second stage of the experiment is concerned with the status of apnea so that only the heartbeat was characterised. In this case, we considered an overall observation time of $56 \mathrm{sec}$ divided in 7 time intervals made of 500 time samples

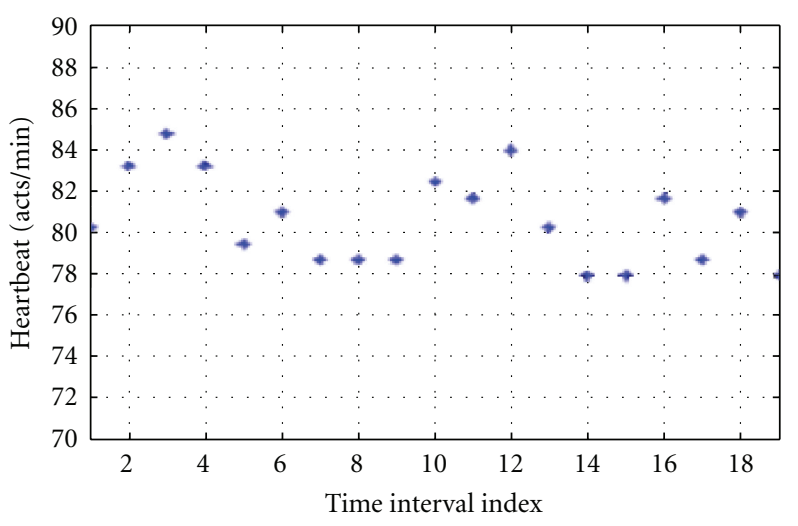

(a)

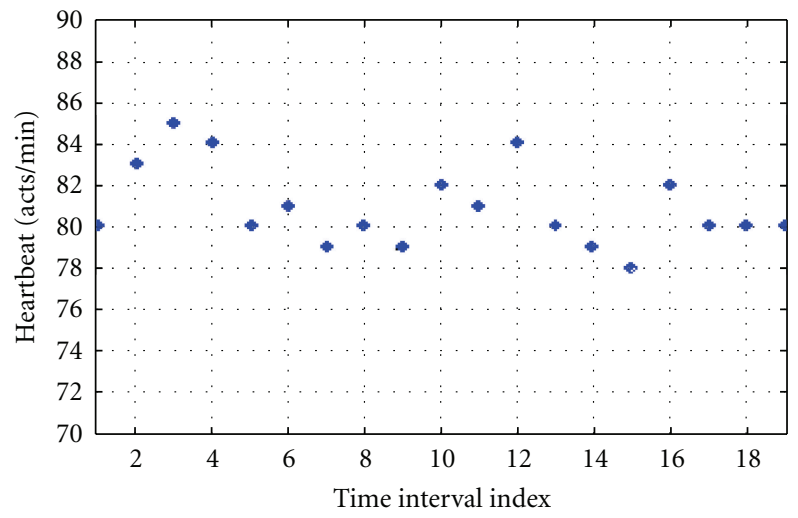

(b)

FIgURE 6: Comparison between the data-processing approaches for the heartbeat. (a) first dataprocessing approach; (b) second dataprocessing approach.

$(8 \mathrm{sec})$. Figure 7 depicts the modulus of the measured signal in the 7 time intervals (time window ranging from 800 to 4300 samples).

The heartbeat frequency behaviour is shown in Figure 8 for the two data-processing approaches, and a very good agreement is observed and it can be noted that the frequency is almost uniform apart from the first two intervals. In particular for the first interval, the lower detected frequency is due to the clearly depicted oscillation, almost at the end of the first time interval, which cannot be associated to heartbeat activity.

\section{Conclusions}

The paper has presented the feasibility study of an approach based on a continuous wave radar for the life signs monitoring. In particular, two different data approaches have been exploited with similar performances, and it has been possible to show the effectiveness of the overall system (hardware plus software) as a reliable tool for a long-term monitoring of breathing and heartbeat activity.

The future activities will address different topics toward the use of the system in full operative conditions in order to account for the obstacle between the radar system and 


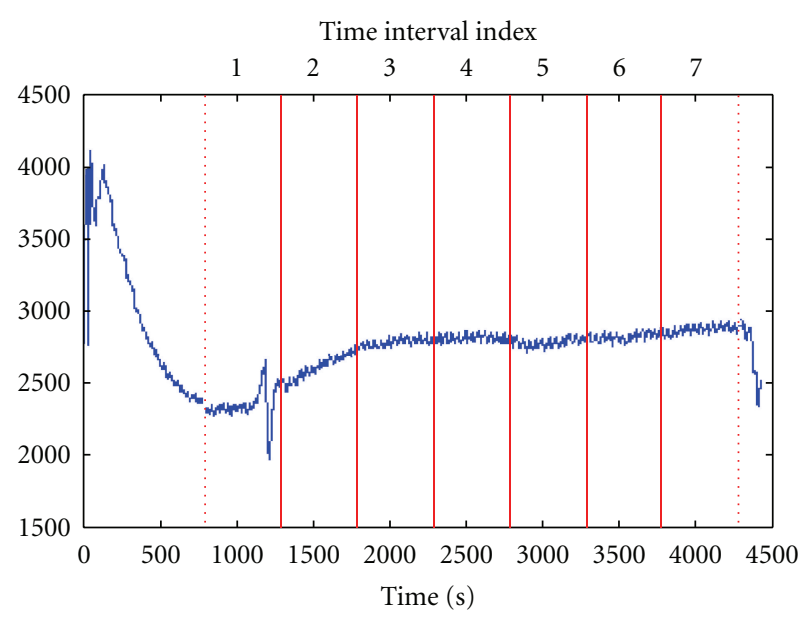

FIGURE 7: Amplitude of the heartbeat signal. The time intervals used in the monitoring are also pointed out.

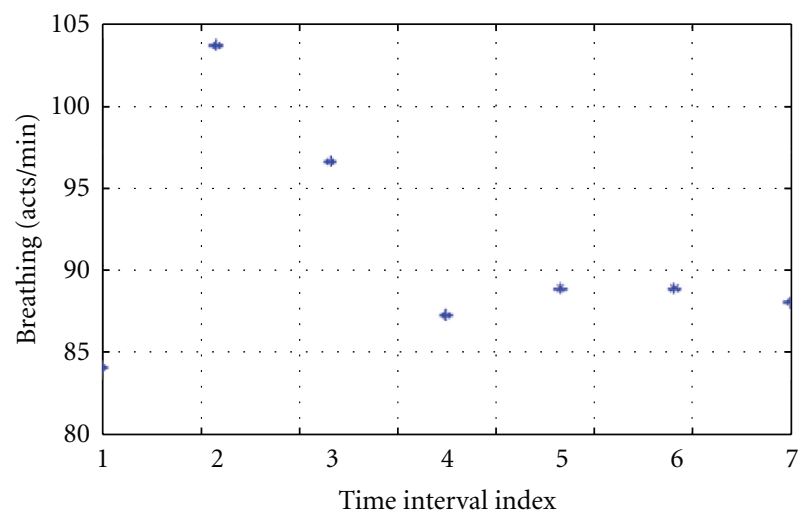

(a)

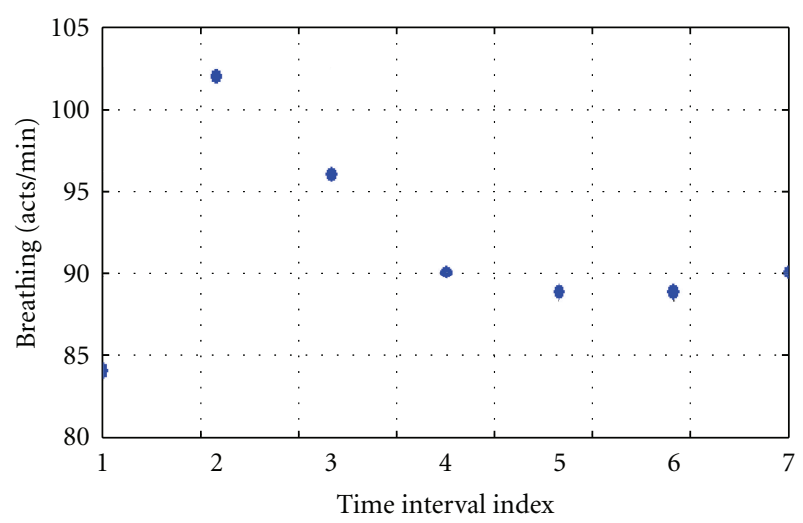

(b)

Figure 8: Comparison between the data-processing approaches for the heartbeat activity: (a) (upper) first data-processing approach; (b) (lower) second data-processing approach.

the target; to characterise target movements different from the life signs; to analyse the radar signal, with the aim to provide a stress level estimation.

The computational cost is suitable to make the processing in a real time (i.e., in few seconds after the data collection) and complies with the necessities of the application frame, which requires fastness of the operations, but more important the reliability of the result. In this frame, we consider both the processing approaches suitable for an implementation in real systems. Furthermore, the crossvalidation between the two proposed approaches can mitigate the possible interpretation ambiguity arisen from the use of a single technique.

\section{Acknowledgment}

This research has been performed in the framework of the "Active and Passive Microwaves for Security and Subsurface imaging (AMISS)" EU 7th Framework Marie Curie Actions IRSES project (PIRSES-GA-2010-269157).

\section{References}

[1] E. J. Baranoski, "Through-wall imaging: historical perspective and future directions," Journal of the Franklin Institute, vol. 345, no. 6, pp. 556-569, 2008.

[2] Z. Yang, J. Xijing, J. Teng, Z. Zhu, L. Hao, and W. Jianqi, "Detecting and identifying two stationary-human-targets: a technique based on bioradar," in Proceedings of the 1st International Conference on Pervasive Computing, Signal Processing and Applications (PCSPA '10), pp. 981-985, September 2010.

[3] K. M. Chen, Y. Huang, J. Zhang, and A. Norman, "Microwave life-detection systems for searching human subjects under earthquake rubble or behind barrier," IEEE Transactions on Biomedical Engineering, vol. 47, no. 1, pp. 105-114, 2000.

[4] S. I. Ivashov, V. V. Razevig, A. P. Sheyko, and I. A. Vasilyev, "Detection of human breathing and heartbeat by remote radar," in Proceedings of the Progress in Electromagnetics Research Symposium (PIERS '04), pp. 663-666, Pisa, Italy, March 2004.

[5] M. Pieraccini, G. Luzi, D. Dei, L. Pieri, and C. Atzeni, "Detection of breathing and heartbeat through snow using a microwave transceiver," IEEE Geoscience and Remote Sensing Letters, vol. 5, no. 1, pp. 57-59, 2008.

[6] E. M. Staderini, "UWB radars in medicine," IEEE Aerospace and Electronic Systems Magazine, vol. 17, no. 1, pp. 13-18, 2002.

[7] L. N. Anishchenko, A. S. Bugaev, S. I. Ivashov, and I. A. Vasiliev, "Application of bioradiolocation for estimation of the laboratory animals' movement activity," PIERS Online, vol. 5, no. 6, pp. 551-554, 2009.

[8] L. Anishchenko, A. Bugaev, S. Ivashov, and A. Zhuravlev, "Bioradar for monitoring of human adaptive capabilities," in Proceedings of the 30th General Assembly and Scientific Symposium of International Union of Radio Science ( URSI '11), Istanbul, Turkey, 2011.

[9] A. S. Bugaev, V. V. Chapursky, and S. I. Ivashov, "Mathematical simulation of remote detection of human breathing and heartbeat by multifrequency radar on the background of local objects reflections," in IEEE International Radar Conference Record, pp. 9-12, Arlington, Va, USA, May 2005.

[10] M. D’Urso, G. Leone, and F. Soldovieri, “A simple strategy for life signs detection via an X-band experimental set-up," Progress in Electromagnetics Research C, vol. 9, pp. 119-129, 2009.

[11] L. N. Anishchenko and V. B. Parashin, "Design and application of the method for biolocation data processing," in Proceedings of the 4th Russian-Bavarian Conference on Biomedical Engineering at Moscow Institute of Electronic Technology (Technical University), pp. 289-294, Moscow, Russia, 2008. 

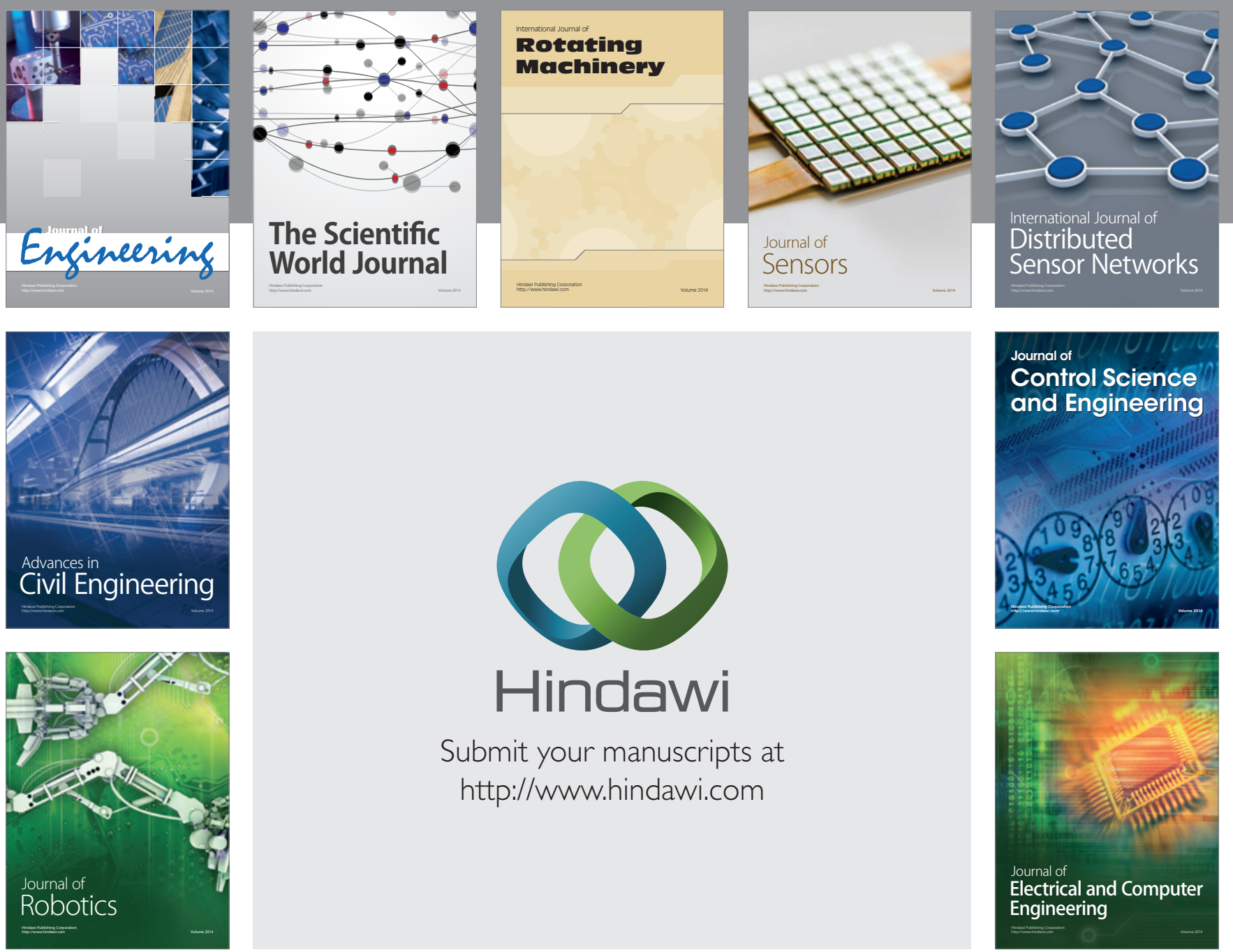

Submit your manuscripts at

http://www.hindawi.com
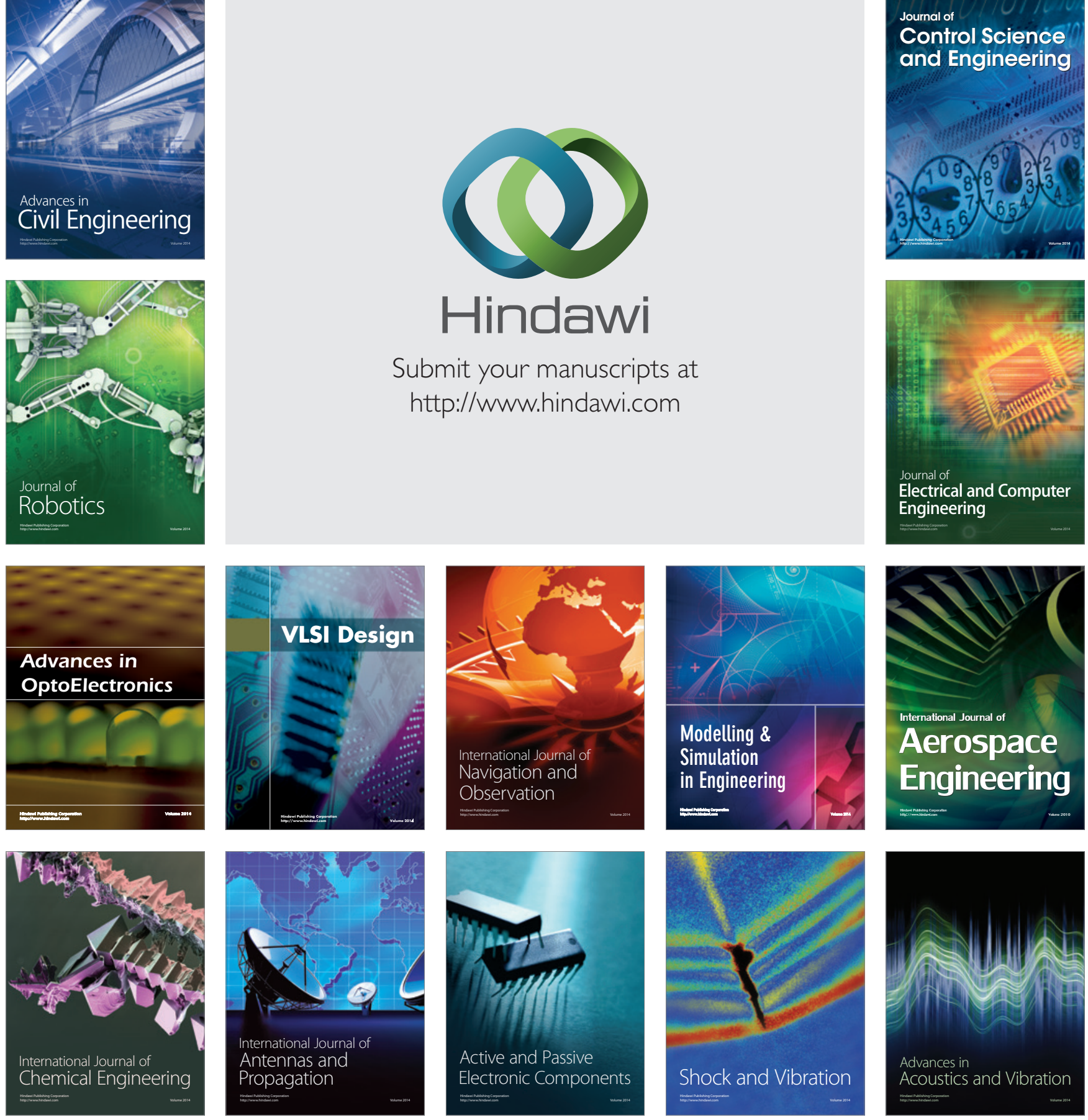